\title{
Escala diagramática para avaliação de severidade de mancha-parda em arroz
}

\author{
Diagrammatic scale for assessment of rice brown spot severity \\ Giuvan Lenz $^{{ }^{*}}$ Ricardo Silveiro Balardin ${ }^{\mathrm{I}}$ Gerson Dalla Corte ${ }^{\mathrm{I}}$ \\ Leandro Nascimento Marques ${ }^{I}$ Daniel Debona ${ }^{I}$
}

\section{RESUMO}

Uma escala diagramática com seis níveis: 1,6 ; 3,2; 6,4; 12,6; 23,1 e 38,6\% foi desenvolvida e validada para quantificar a severidade da mancha-parda em arroz causada por Bipolaris oryzae. A escala considerou o limite de severidade máxima da doença observado no campo,e os níveis intermediários seguiram incrementos logarítmicos, obedecendo-se à "Lei do estímulo de Weber-Fechner". Inicialmente, a estimativa da severidade foi realizada sem auxílio da escala, em 30 folhas com diferentes níveis de severidade, por oito indivíduos, sem experiência na avaliação de doenças. Em seguida, os mesmos avaliadores utilizaram a escala diagramática proposta. As avaliações com a escala diagramática foram mais precisas e acuradas nas estimativas de todos os avaliadores, não ocorrendo erro sistemático na superestimativa ou subestimativa da doença entre estes. A escala diagramática proposta foi considerada adequada para estimar a severidade da mancha-parda em arroz.

Palavras-chave: Bipolaris oryzae, Drechslera oryzae, diagnose, mancha foliar, fitopatometria.

\section{ABSTRACT}

A diagrammatic scale with six levels of disease severity: $1.6 ; 3.2 ; 6.4 ; 12.6 ; 23.1$ and $38.6 \%$ was developed and validated to assess rice brown spot, caused by Bipolaris oryzae. The scale was developed considering the maximum limit of disease severity observed in the field and the intermediate values followed logarithmic increases according to the "Stimulus Law by Weber-Fechner". Initially, the estimatives of severity were performed without the use of the scale in 30 leaves with different levels of severity by eight people without previous experience in assessing diseases severity. Then, the same people estimated the severity of leaves using the proposed diagrammatic scale. Assessments with the diagrammatic scale were of great precision for all raters and did not presented systematic errors in over- or underestimating the disease severity. The proposed diagrammatic scale was considered adequate to estimate rice brown spot severity.

Key words: Bipolaris oryzae, Drechslera oryzae, diagnosis, leaf spot, phytopatometry.

\section{INTRODUÇÃO}

A mancha-parda, causada pelo fungo Bipolaris oryzae (sin. Drechslera oryzae), é uma das doenças mais importantes da cultura do arroz irrigado no Brasil (PRABHU \& FILIPPI, 1997) e na maioria dos países produtores desse cereal. Em boa parte das lavouras orizícolas do planeta, apenas a brusone (Pyricularia grisea) apresenta maior importância em relação à mancha-parda (OU, 1985).

A simples observação da ocorrência ou a avaliação da doença utilizando-se critérios subjetivos, na maioria das vezes, induz erro, seja na avaliação da resistência de cultivares, seja na tomada de decisão de controle, sendo assim necessária a adoção de critérios precisos na quantificação de doenças (LENZ et al., 2009).

A utilização de escalas diagramáticas pode reduzir a subjetividade das estimativas de severidade entre os avaliadores, melhorando a acurácia e a precisão da avaliação (MARTINS et al., 2004; AQUINO et al., 2008; LENZ et al., 2009). No entanto, as escalas precisam ser reproduzíveis e devem apresentar níveis suficientes

IDepartamento de Defesa Fitossanitária, Universidade Federal de Santa Maria (UFSM), 97105-900, Santa Maria, RS, Brasil. Email: giuvanlenz@gmail.com.*Autor para correspondência. 
para a caracterização do progresso da doença (GODOY et al., 2006).

Na elaboração de escalas diagramáticas, devem ser considerados aspectos como o limite superior da escala, que deve corresponder à intensidade máxima de doença observada no campo, e a determinação da intensidade real de doença no campo e sua representação na escala devem ter alta precisão. Os níveis intermediários da escala devem respeitar as limitações da acuidade visual humana definidas pela lei de estímulo-resposta de Weber-Fechner, na qual a acuidade visual é proporcional ao logaritmo da intensidade do estímulo (HORSFALL \& COWLING, 1978). Após a elaboração, os níveis de precisão e acurácia das estimativas propiciados pela utilização da escala diagramática devem ser analisados para determinar a qualidade das estimativas (KRANZ, 1988).

Considerando a inexistência de um método que auxilie na estimativa da porcentagem de área foliar com sintomas de mancha-parda em arroz, este trabalho teve como objetivos desenvolver uma escala diagramática para avaliação da severidade da doença e analisar os níveis de acurácia e precisão das estimativas geradas a partir de sua utilização.

\section{MATERIAL E MÉTODOS}

\section{Elaboração da escala diagramática \\ Para elaboração da escala} diagramática, foram coletadas 100 folhas de arroz com diferentes níveis de severidade de manchaparda, sendo individualmente digitalizadas por meio de scanner. As imagens foram então transferidas para um microcomputador, com resolução de 300dpi. Em seguida, a severidade foi estimada por meio do software Quant ${ }^{\circledR}$ (VALE et al., 2003), servindo de base para avaliar a precisão e acurácia da escala. Os intervalos da escala foram calculados com o auxílio do programa 2 LOG $^{\circledR}$ (TOVAR-SOTO et al., 2002).

Baseando-se na lei de Weber-Fechner de acuidade visual (HORSFALL \& COWLING, 1978), bem como na forma, distribuição e frequência das lesões, foi confeccionada, com a ajuda do editor gráfico CorelDRAW ${ }^{\circledR}$ (Corel), uma escala diagramática logarítmica com seis níveis de severidade (Figura 1) e nela representados os sintomas de cada nível de severidade, procurando-se manter os padrões de distribuição das lesões.
Validação da escala diagramática

Para validação da escala diagramática proposta, foram selecionadas 30 folhas com diferentes níveis de severidade e apresentadas a oito avaliadores sem experiência prévia na avaliação de doenças. Os avaliadores estimaram em um primeiro momento a severidade da mancha-parda do arroz sem a escala diagramática e, em seguida, estes repetiram a avaliação com o auxílio desta.

Regressões lineares entre a severidade real (variável independente), fornecida pelo software Quant ${ }^{\circledR}$, e a estimada (variável dependente) pelos avaliadores foram calculadas para cada um deles (Figura 2). A acurácia das estimativas de cada avaliador foi determinada pelo teste $t$ aplicado às estimativas dos parâmetros de interseção da regressão linear ( $a$ ), para verificar a hipótese $\mathrm{H}_{0}: a=0$, e coeficiente angular da reta $(b)$, para testar a hipótese H0: $b=1$, ao nível de probabilidade $\mathrm{P}=0,05$. A precisão foi estimada pelo coeficiente de determinação da regressão $\left(R^{2}\right)$ e pela variação dos resíduos expressa pela severidade

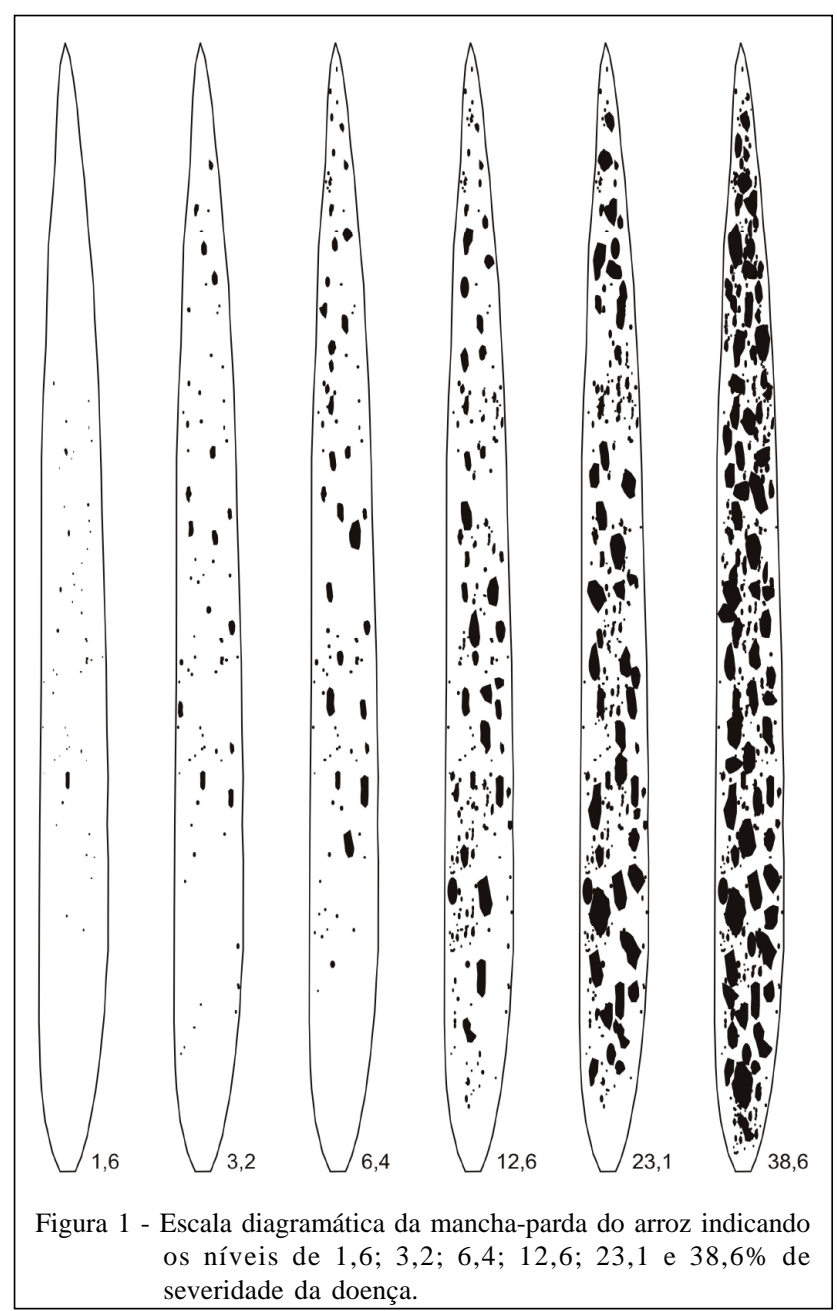

Ciência Rural, v.40, n.4, abr, 2010. 


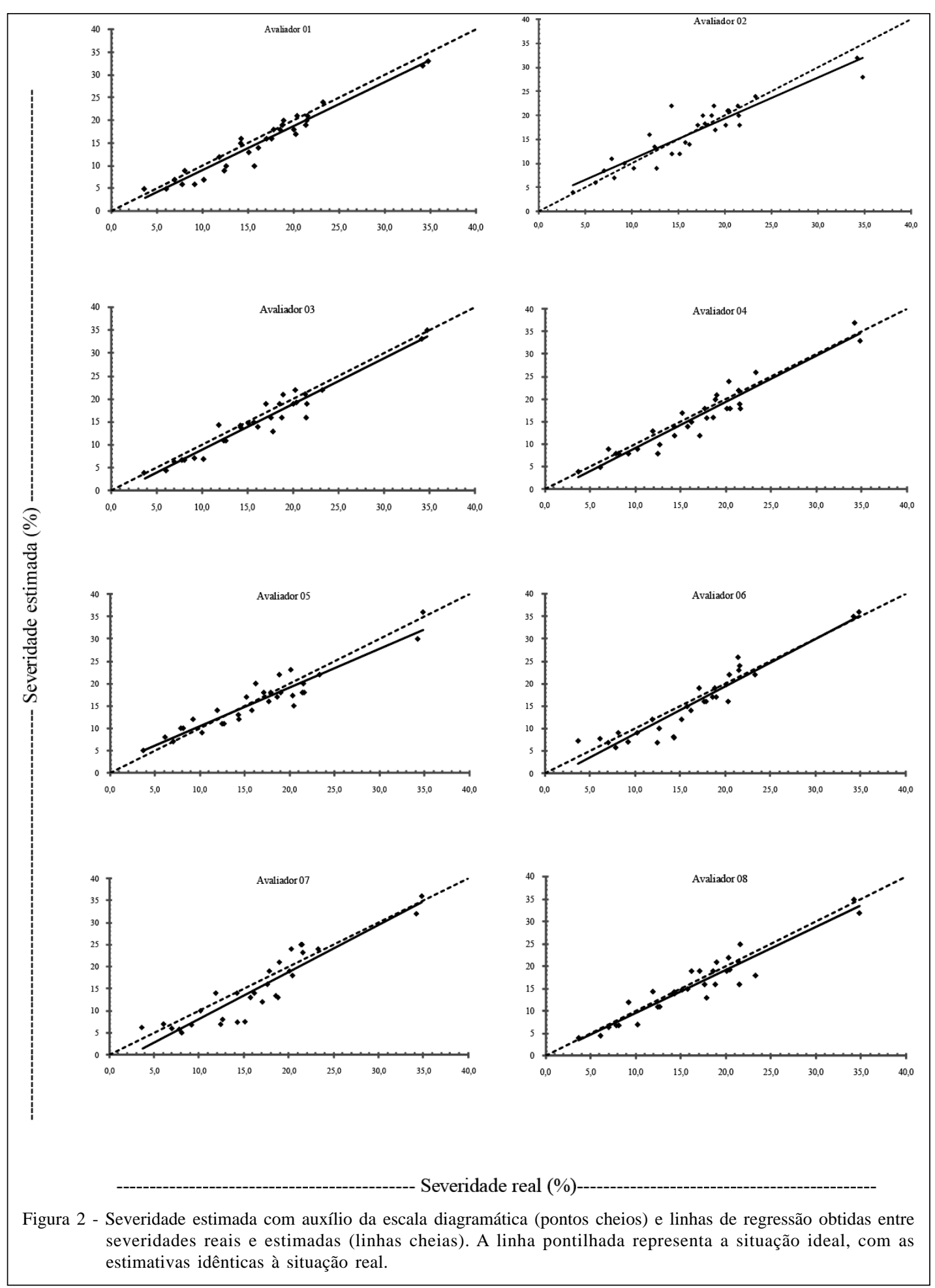

estimada menos a severidade real (Figuras 3 e 4). A análise estatística foi realizada com o software STATÍSTICA $^{\circledR}$ (StatSoft), e os gráficos foram desenvolvidos com o auxílio do software Excel $2007^{\circledR}$ (Microsoft Corporation).

\section{RESULTADOS E DISCUSSÃO}

A escala elaborada apresentou como limite superior de severidade o valor de $38,6 \%$, enquanto que o valor mínimo foi de $1,6 \%$. Considerando esses limites, 


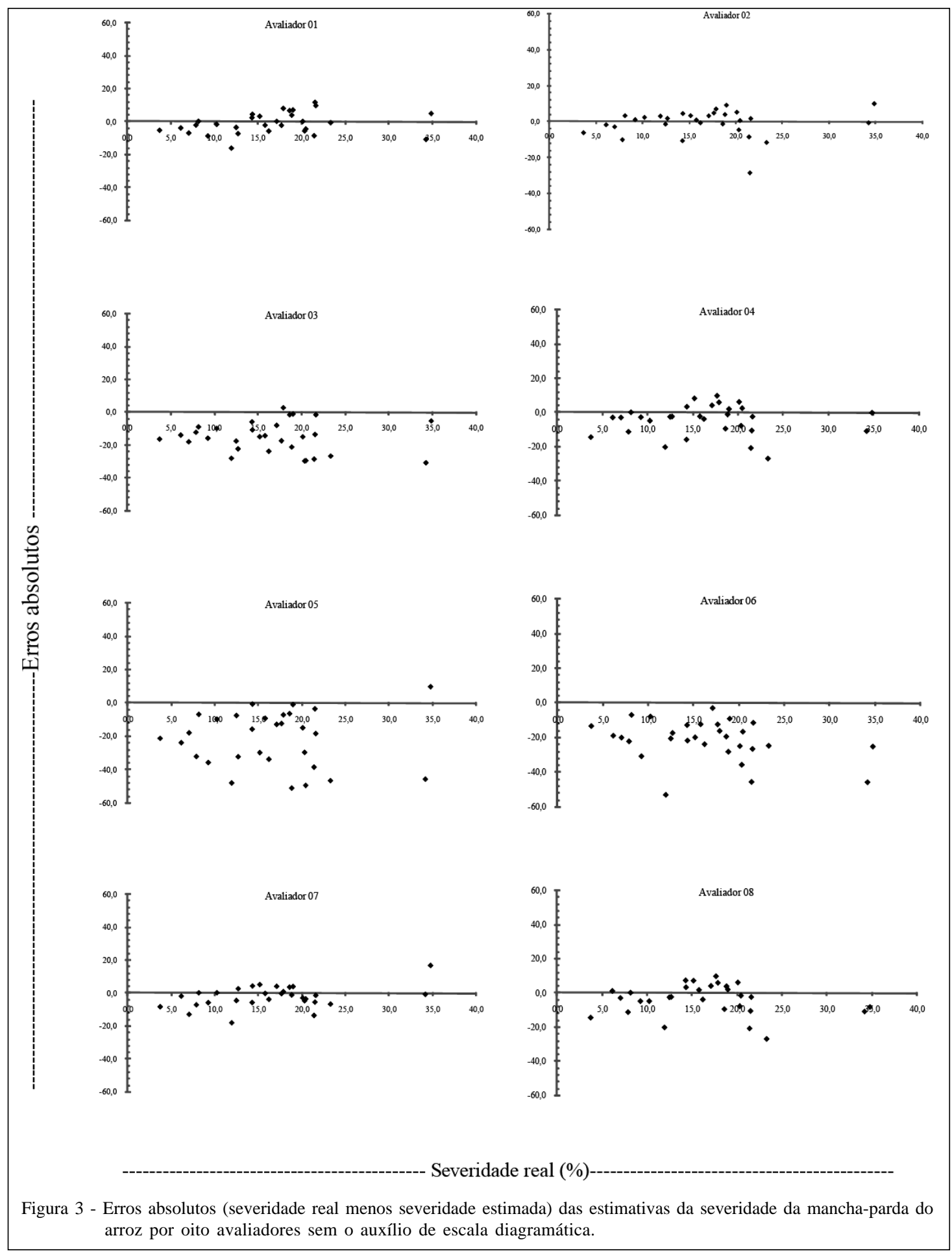

a escala diagramática desenvolvida para a quantificação da doença foi constituída de seis níveis de severidade: 1,6; 3,2; 6,4; 12,6; 23,1 e 38,6\% da área foliar com sintomas da doença (Figura 1).

A comparação dos dados das análises de regressão dos avaliadores, com e sem o auxílio da escala diagramática, demonstrou que a escala foi efetiva para aumentar a acurácia e precisão nas avaliações da doença (Tabela 1). A precisão indica a confiabilidade nas avaliações de doença e pode ser medida pelo coeficiente de determinação da regressão $\left(\mathrm{R}^{2}\right)$ entre as severidades real e estimada, a variância dos erros 


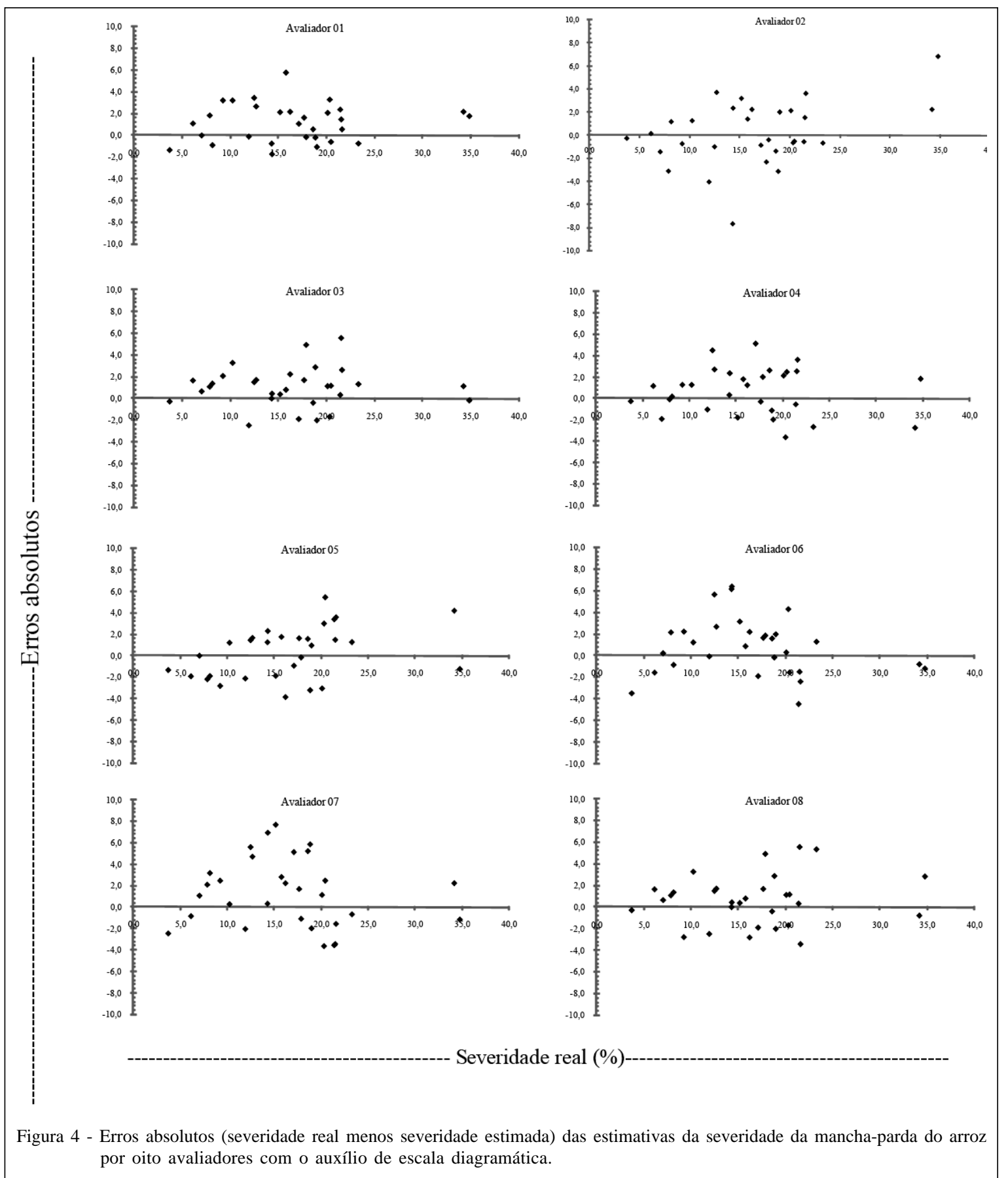

absolutos e a repetibilidade das estimativas por um mesmo avaliador (NUTTER JUNIOR et al., 1993; MICHEREFF et al., 2000). Já a acurácia se refere à proximidade entre a severidade estimada e a real e pode ser medida comparando-se os parâmetros "a" e "b" com os valores zero e um respectivamente, em que valores de "a” significativamente diferentes de zero indicam desvios constantes e valores de coeficiente angular (b) significativamente diferentes de um indicam haver desvios sistemáticos (VALE et al., 2004; BELASQUE JÚNIOR et al., 2005).

A validação de uma escala é realizada para aferir se ela está apta a ser utilizada a campo nas mensurações de severidade por pesquisadores e produtores. Neste trabalho, as análises para validação da escala diagramática mostraram que os avaliadores 
Tabela 1 - Acurácia e precisão representadas pela interseção $(a)$, pela inclinação $(b)$ e pelo coeficiente de determinação $\left(R^{2}\right)$ nas avaliações da severidade da mancha-parda do arroz, causada por Bipolaris oryzae, sem e com o auxílio da escala diagramática.

\begin{tabular}{|c|c|c|c|c|c|c|c|c|c|}
\hline \multirow[t]{2}{*}{ Coeficiente } & \multicolumn{9}{|c|}{ Avaliadores } \\
\hline & 01 & 02 & 03 & 04 & 05 & 06 & 07 & 08 & Média \\
\hline$a$ & 4,77 & 2,18 & $12,97^{*}$ & 4,07 & $21,40^{*}$ & $12,78^{*}$ & $8,30^{*}$ & 0,87 & 8,42 \\
\hline$b$ & $0,78^{*}$ & 0,92 & 1,14 & 1,02 & 1,02 & $1,53^{*}$ & $0,62^{*}$ & $1,17^{*}$ & 1,03 \\
\hline$R^{2}$ & 0,44 & 0,43 & 0,43 & 0,41 & 0,16 & 0,49 & 0,36 & 0,46 & 0,40 \\
\hline$a$ & $-0,77$ & 2,30 & $-0,98$ & $-1,02$ & 1,85 & $-1,81$ & $-2,37$ & $-0,04$ & 1,39 \\
\hline$b$ & 0,97 & 0,85 & 0,99 & 1,02 & 0,86 & 1,05 & 1,06 & 0,96 & 0,97 \\
\hline$R^{2}$ & 0,94 & 0,84 & 0,94 & 0,92 & 0,89 & 0,89 & 0,85 & 0,89 & 0,90 \\
\hline
\end{tabular}

* representa situações em que a hipótese de nulidade ( $a=0$ ou $b=1)$ foi rejeitada pelo teste $t(\mathrm{P}<0,05)$.

que utilizaram a escala apresentaram boa precisão nas suas estimativas para as equações de regressão estabelecidas entre a severidade real e a estimada, com coeficiente de determinação médio de 0,89 e valores variando entre 0,84 e 0,94 . Resultados semelhantes foram encontrados por GODOY et al. (2006), que obtiveram coeficiente de determinação médio de 0,86 com o uso de escala na avaliação da ferrugem asiática da soja. Contudo, sem o auxílio da escala, os avaliadores foram pouco precisos, com coeficiente de determinação médio de 0,39, com mínimo de 0,16 e máximo de 0,49. Esses valores demonstram que, com o uso da escala, as estimativas visuais de severidade foram sistematicamente relacionadas com os valores reais. Diversos estudos já confirmaram o aumento na precisão das avaliações visuais de severidade com a utilização de escalas diagramáticas (BARBOSA et al., 2006; GODOY et al., 2006; LENZ et al., 2009), demonstrando a importância dessa ferramenta em estudos de quantificação de doenças.

Na primeira etapa da validação da escala diagramática, ou seja, sem a utilização desta, os valores do intercepto de quatro avaliadores (03, 05, 06 e 07) diferiram estatisticamente de zero. Considerando a média dos avaliadores, o valor do intercepto foi de 8,42 (Tabela 1), sinalizando que houve uma tendência de superestimação da severidade de mancha-parda. Em contrapartida, com a utilização da escala, todos os avaliadores apresentaram valores de $a$ estatisticamente iguais a zero, com valor médio de 1,39 , bem menor daquele observado na ausência da escala. Todos os valores do coeficiente angular da reta dos avaliadores não diferiram significativamente de 1 , independente do uso ou não da escala diagramática.

Outra forma de avaliar a precisão é pela distribuição dos erros absolutos. A acurácia pode ser avaliada pela proximidade das equações de regressão entre as severidades real e estimada com a situação ideal (BELASQUE JÚNIOR et al., 2005). Autilização da escala promoveu a redução dos erros absolutos, quando comparada com a distribuição dos resíduos das estimativas observados sem escala diagramática (Figuras 3 e 4).

Os erros absolutos observados para as estimativas obtidas por todos os avaliadores, com o auxílio da escala, ficaram concentrados dentro da faixa de $\pm 10 \%$, valores considerados adequados segundo critérios adotados por programas de treinamento na quantificação de doenças, como o Distrain ${ }^{\circledR}$ (TOMERLIN \& HOWELL, 1988) e Disease Pro ${ }^{\circledR}$ (NUTTER JUNIOR \& WORAWITLIKIT, 1989), os quais classificam como excelente um avaliador cujos erros não ultrapassam $5 \%$ e bom aquele cujos erros sejam menores que $10 \%$. De outra forma, a distribuição dos resíduos das avaliações realizadas sem o auxílio da escala diagramática apresentou estimativas com erros absolutos variando entre -53,1 (Figura 3, avaliador 06) e 16,8 (Figura 3, avaliador 07), com a média dos erros (em módulo) de todos os avaliadores igual a 11,16. Quando a avaliação foi realizada com o auxílio da escala, todos os avaliadores melhoraram sua precisão e reduziram os erros sistemáticos das estimativas, sendo a média dos erros (em módulo) de todos os avaliadores igual a 2,04. STONEHOUSE (1994) destacou que a presença de algum nível de erro absoluto nas mensurações pode ser compensada pela rapidez e padronização, que resulta no uso de escalas diagramáticas.

A diferença entre os avaliadores na determinação da severidade de mancha-parda na cultura do arroz corrobora as observações feitas por NUTTER JUNIOR \& SCHULTZ (1995) em relação à habilidade humana diferenciada para discriminar níveis de doença. A qualidade da estimativa visual de uma 
determinada doença é influenciada não apenas por estímulos psicológicos, mas também por fatores, como a complexidade da amostra, o tamanho e a forma das lesões, a cor e o número (KRANZ, 1988), além da dificuldade de o avaliador manter o foco na avaliação (SHOKES et al., 1987). O desenvolvimento de sistemas padronizados de avaliação de doenças no arroz é de grande responsabilidade, pois estimativas visuais imprecisas levam a erros que alteram conclusões de ensaios (PARKER et al., 1995).

\section{CONCLUSÃO}

Os níveis de acurácia, precisão e reprodutibilidade das mensurações da severidade da mancha-parda em arroz melhoram com o uso da escala diagramática, demonstrando ser esta uma ferramenta útil para avaliações de severidade mesmo por pessoas desprovidas de treinamento ou experiência prévia em quantificação de doenças.

\section{REFERÊNCIAS}

AQUINO, L.A. et al. Elaboração e validação de escala diagramática para quantificação da mancha de ramularia do algodoeiro. Summa Phytopathologica, v.34, p.361-363, 2008. Disponível em: <http:/ / w w w. s c i e l o.b r / s c i e lo.ph p ? p i d = S 0100 54052008000400012\&script $=$ sci_arttext\&tlng $=$ target $=$ _blank $>$. Acesso em: 11 mar. 2009. doi: 10.1590/S0100-54052008000400012.

BARBOSA, M.A.G. et al. Elaboração e validação de escala diagramática para avaliação da severidade da ferrugem branca do crisântemo. Summa Phytopathologica, v.32, p.57-62, 2006. Disponível em: <http://www.scielo.br/scielo.php?pid=S010054052006000100008\&script=sci_arttext $>$. Acesso em: 10 mar. 2009. doi: 10.1590/S0100-54052006000100008.

BELASQUE JÚNIOR, J. et al. Escalas diagramáticas para avaliação da severidade do cancro cítrico. Fitopatologia Brasileira, v.30, p.387-393, 2005. Disponível em: <http:// www.scielo.br/scielo.php?script=sci_arttext\&pid=S0100$41582005000400008 \& \operatorname{lng}=$ pt $\& n r m=$ iso $\&$ tlng $=$ pt $>$. Acesso em: 10 mar. 2009. doi: 10.1590/S0100-41582005000400008.

GODOY, C.V. et al. Diagrammatic scale for assessment of soybean rust severity. Fitopatologia Brasileira, v.31, p.6368, 2006. Disponível em: <http://www.scielo.br/ scielo.php? script =sci_arttext\&pid=S010041582006000100011>. Acesso em: 11 mar. 2009. doi: 10.1590/S0100-41582005000400008.

HORSFALL, J.G.; COWLING, E.B. Pathometry: the measurement of plant disease. In: __________ Plant disease: an advanced treatise - how disease develops in populations. New York: Academic, 1978. p.119-136.

KRANZ, J. Measuring plant disease. In: KRANZ, J.; ROTEM, J. Experimental techniques in plant disease epidemiology. Heidelberg: Springer-Verlag, 1988. p.35-50.

LENZ, G. et al. Elaboração e validação de escala diagramática para quantificação da mancha de isariopsis da videira. Ciência
Rural, v.39, n.8, p.2301-2308, 2009. Disponível em: <http:/ /www.scielo.br/scielo.php?script=sci_arttext\&pid=S010384782009000800005>. Acesso em: 15 mar. 2009. doi: 10.1590/S0103-84782009000800005.

MARTINS, M.C. et al. Escala diagramática para quantificação do complexo de doenças foliares de final de ciclo em soja. Fitopatologia Brasileira, v.29, p.179-184, 2004. Disponível em: $<$ http://www.scielo.br/scielo.php?script=sci_arttext\&pid=S010041582004000200009\&lng=pt\&nrm=iso>. Acesso em: 09 mar. 2009. doi: 10.1590/S0100-41582004000200009.

MICHEREFF, S.J. et al. Escala diagramática para avaliação da queima das folhas do inhame. Fitopatologia Brasileira, v.25, p.612-619, 2000.

NUTTER JUNIOR., F.W.; SCHULTZ, P.M. Improving the accuracy and precision of disease assessments: selection of methods and use of computer-aided training programs. Canadian Journal of Plant Pathology, v.17, p.174-184, 1995.

NUTTER JUNIOR, F.W. et al. Assessing the accuracy, intrarater repeatability, and inter-rater reliability of disease assessment systems. Phytopathology, v.83, p.806-812, 1993.

NUTTER JUNIOR, F.W.; WORAWITLIKIT, O. Disease Pro: a computer program for evaluating and improving a person ability to assess disease proportion. Phytopathology, v.79, p.1135. 1989.

OU, S.H. Rice diseases. 2.ed. Slough: CAB International Mycological Institute, 1985. 380p.

PARKER, S.R. et al. The reliability of visual estimates of disease severity on cereal leaves. Plant Pathology, v.44, p.856-864, 1995.

PRABHU, A.S.; FILIPPI, M.C. Arroz (Oryza sativa L.) Controle de doenças. In: VALE, F.X.R.; ZAMBOLIM, L. Controle de doenças de plantas: grandes culturas. Viçosa: Universidade Federal de Viçosa, 1997. p.51-79.

SHOKES, F.M. et al. Reliability of disease assessment procedures: a case study with late leaf spot of peanut. Oléagineux, v.42, p.245-251, 1987.

STONEHOUSE, J. Assessment of Andean bean diseases using visual keys. Plant Pathology, v.43, p.519-527, 1994.

TOMERLIN, J.R.; HOWELL, T.A. Distrain: a computer program for training people to estimate disease severity on cereal leaves. Plant Disease, v.72, p.455-459, 1988.

TOVAR-SOTO, A. et al. Escala logarítimica diagramática de severidad de la mancha negra (Colletotrichum gloeosporioides Penz.) en Chirimoyo (Annona cherimola Mill). Revista Mexicana de Fitopatología, v.20, p.103-109, 2002.

VALE, F.X.R. et al. QUANT: a software plant disease severity assessment. INTERNATIONAL CONGRESS OF PLANT PATHOLOGY, 8., 2003, Christchurch Proceedings... Christchurch New Zealand: Plant Pathology Society, 2003. p.105.

VALE, F.X.R. et al. Quantificação de doenças e do crescimento do hospedeiro. In: VALE, F.X.R. et al. Epidemiologia aplicada ao manejo de doenças de plantas. Belo Horizonte: Perfil, 2004. p.91-126. 